\title{
HOMEOMORPHISMS ON A SOLID TORUS
}

\author{
D. R. McMillaN, JR.
}

1. Introduction. An orientable 3-manifold with boundary can be represented as a solid torus $H$ ("cube with handles") plus a disjoint collection of 3-cells attached to the boundary of $H$ along annuli. ${ }^{1}$ Hence, a natural (but difficult) approach to the study of such 3manifolds is to associate with each a system of disjoint simple closed curves in the boundary of a solid torus. It would be useful (e.g., in proving that two 3-manifolds are homeomorphic) to have conditions under which one such system of curves is topologically equivalent to another.

A special case of this problem is considered here. Let $H$ be a solid torus of genus $n$ and let $J$ and $J^{*}$ be two collections of $n$ disjoint simple closed curves in the boundary of $H$. The result is that if each of the collections "generates" $\pi_{1}(H)$ (see $\$ 2$ ), then the collections are topologically equivalent. Some care must be exercised in producing the homeomorphism, since not every homeomorphism on the boundary of $H$ which throws one collection onto the other can be extended to $H$.

2. Preliminaries. Two simplicial complexes will be called equivalent if they have rectilinear subdivisions which are isomorphic complexes. An $n$-cell ( $n$-sphere) is a complex equivalent to an $n$-simplex (boundary of an $n+1$-simplex, respectively). If the closed star of each vertex in the complex $M$ is equivalent to an $n$-cell, then $M$ is by definition an $n$-manifold. The union of those simplexes of $M$ each of whose links is not a sphere is the boundary of $M(\mathrm{Bd} M)$ and the interior of $M$ (Int $M$ ) is $M-\mathrm{Bd} M$. All the manifolds considered here are to be compact. A compact manifold with null boundary is closed.

A continuous mapping between complexes is piecewise linear if it is simplicial relative to some rectilinear subdivision of each. A closed subset $P$ of a complex $K$ is polyhedral if the inclusion map $i: P \rightarrow K$ is piecewise linear for some triangulation of $P$. All mappings employed here are understood to be piecewise linear unless stated otherwise ("piecewise linear" is sometimes used explicitly for emphasis). Regu-

Presented to the Society, April 14, 1962; received by the editors March 15, 1962.

1 To see this, note that since the 3-manifold $M$ has nonempty boundary, it contracts [4] into a 2-complex $K$ which lies in the 2-skeleton of $M$. A "small" regular neighborhood of $K$ in $M$ clearly is of the form described above and, by Theorem 23 of [4], is equivalent to $M$. In case $M$ is contractible, we may (as in our Theorem) take the number of annuli equal to the genus of $H$. 
lar neighborhoods of subcomplexes of manifolds are understood in the sense of Whitehead [4].

A solid torus of genus $n \geqq 0$ is a 3 -manifold $M$ equivalent to the regular neighborhood in $S^{3}$ of a finite, connected 1-dimensional complex with first Betti number $n$. The boundary of $M$ is a closed orientable surface of genus $n$. We write $g(M)=n$.

If $f$ and $g$ are mappings of $I=[0,1]$ into a space $X$ such that $f(1)=g(0)$, let $f \times g$ denote the mapping of $I$ into $X$ given by $(f \times g)(t)$ $=f(2 t)$ for $0 \leqq t \leqq 1 / 2$ and $(f \times g)(t)=g(2 t-1)$ for $1 / 2 \leqq t \leqq 1$. Let $\bar{f} \mathrm{de}-$ note the mapping given by $\bar{f}(t)=f(1-t)$ for $0 \leqq t \leqq 1$. If $f(0)=f(1)=p$, then $[f]$ is the element of $\pi_{1}(X, p)$ determined by $f$.

Let $H$ be a solid torus of genus $n$ and $J_{1} \cdots J_{n}$ disjoint polyhedral simple closed curves on $\mathrm{Bd} H$. Select piecewise linear mappings $f_{1}, \cdots, f_{n}$, where $f_{i}: I \rightarrow J_{i}, f_{i}(0)=f_{i}(1)$, and $f_{i} \mid(0,1)$ is a homeomorphism (the property to follow will not depend upon this selection). If there exists a point $p$ in $\mathrm{Bd} H$ and piecewise linear mappings $\sigma_{1}, \cdots, \sigma_{n}$ where $\sigma_{i}: I \rightarrow \operatorname{Bd} H$ such that $\sigma_{i}(0)=p, \sigma_{i}^{-1}\left(J_{i}\right)=\{1\}$, $\sigma_{i}(1)=f_{i}(0)=f_{i}(1), \sigma_{i}(I) \cap J_{j}=\varnothing$ for $i \neq j$, and the elements $\left[\left(\sigma_{1} \times f_{1}\right) \times \bar{\sigma}_{1}\right], \cdots,\left[\left(\sigma_{n} \times f_{n}\right) \times \bar{\sigma}_{n}\right]$, generate $\pi_{1}(H, p)$, then it will be said that $J_{1}, \cdots, J_{n}$, generate $\pi_{1}(H)$ or form a set of generators for $\pi_{1}(H)$. Two sets of generators are equivalent if there is a piecewise linear homeomorphism of $H$ onto $H$ throwing the elements of one collection onto those of the other.

The following result seems to be generally known, and is an easy consequence of the Loop Theorem [1] and Dehn's Lemma [2].

LEMMA. Let $M$ be a compact 3-manifold with connected boundary of genus $n$. If $M$ can be embedded in $E^{3}$ and $\pi_{1}(M)$ is a free group, then $M$ is a solid torus and $g(M)=n$.

3. The Theorem. We show the following:

TheOREM. Let $H$ be a solid torus with $g(H)=n$. Then, any two sets of generators for $\pi_{1}(H)$ are equivalent.

Proof. Let $J_{1}, \cdots, J_{n}$ generate $\pi_{1}(H)$. We show first that, if $n \geqq 2$, there is a polyhedral 2-cell $D$ in $H$ such that $D \cap \operatorname{Bd} H=\operatorname{Bd} D$, $\bigcup_{i=1}^{n} J_{i} \cap D=\varnothing$, and $D$ separates the $J_{i}$ 's nontrivially in $H$. The remainder of the argument will then be an easy induction.

To do this, let $\rho$ be a combinatorial isomorphism of $H$ onto $H^{*}$, a disjoint copy. Let $N\left(J_{1}, \mathrm{Bd} H\right), \cdots, N\left(J_{n}, \mathrm{Bd} H\right)$ be disjoint regular neighborhoods in $\mathrm{Bd} H$ of the $J_{i}$ 's. Form the compact 3manifold $M$ by identifying points which correspond under 
$\rho \mid \operatorname{Bd} H-\bigcup_{i=1}^{n}$ Int $N\left(J_{i}\right.$, Bd $\left.H\right)=S^{\prime}$. Let $\eta: H \cup H^{*} \rightarrow M$ be the identification map, and let $S=\eta\left(S^{\prime}\right)$.

Note that Bd $M$ consists of closed surfaces (2-manifolds) of genus one: $T_{1}, \cdots, T_{n}$. Since $\pi_{1}(H)$ is free of rank $n$ and is generated by $n$ loops in $S^{\prime}$, it follows from van Kampen's Theorem [3] that $\pi_{1}(M)$ is freely generated by $n$ loops in $S$. For future reference, notice that each of these loops determines a conjugate class of elements in $\pi_{1}(M)$ which coincides with exactly one of the classes determined by $\eta\left(J_{1}\right), \cdots, \eta\left(J_{n}\right)$. Hence $M$ could be embedded in a simply-connected 3-manifold by attaching one solid torus to each $T_{i}$ in such a way that each $\eta\left(J_{i}\right)$ becomes null-homotopic. Thus each closed surface in Int $M$ separates $M$.

Consider the inclusion homomorphism $i^{*}: \pi_{1}\left(T_{1}\right) \rightarrow \pi_{1}(M)$. Since $\pi_{1}(M)$ is a free group and $\pi_{1}\left(T_{1}\right)$ is not, the kernel of $i^{*}$ is nontrivial. By [1] and [2], there is a polyhedral 2-cell $\Delta$ in $M$ such that $\Delta \cap B d M$ $=\Delta \cap T_{1}=\mathrm{Bd} \Delta$ and $\mathrm{Bd} \Delta$ does not separate $T_{1}$. A regular neighborhood in $M$ of $\Delta \cup T_{1}$ has a 2 -sphere $K^{\prime}$ as one boundary component and $K^{\prime}$ lies in Int $M$ and separates $T_{1}$ from $\bigcup_{i=2}^{n} T_{i}$. Thus, there is a polyhedral 2-sphere $K^{\prime}$ which (1) lies in Int $M,(2)$ is in general position relative to $S$, and (3) separates the $T_{i}$ 's nontrivially in $M$. Let $K$ be a 2 -sphere with these three properties and with the number $m$ of components of $K \cap S$ (these are simple closed curves) minimal. By (3), $m \neq 0$.

No component of $K \cap S$ can bound a 2-cell on $\eta(\mathrm{Bd} H)$ or on $\eta\left(\mathrm{Bd} H^{*}\right)$. If this were to occur, there would be a 2 -cell $E \subseteq S$ such that Bd $E \subseteq K$ and $K \cap$ Int $E=\varnothing$. Then by splitting $K \cup E$ along $E$ one obtains 2 -spheres $K_{0}, K_{1}$, lying in the union of $K$ with a small neighborhood of $E$, each having properties (1) and (2) above but meeting $S$ in fewer than $m$ simple closed curves. Hence $K_{0}$ and $K_{1}$ must fail to have property (3), which implies that $K$ does not have property (3), a contradiction.

Now we show how to choose the required $D$. Let $D^{\prime}$ be a polyhedral 2 -cell in $K$ such that Bd $D^{\prime} \subseteq S$ and $S \cap$ Int $D^{\prime}=\varnothing$. Since $S \cap I n t D^{\prime}$ $=\varnothing$, assume (say) that Int $D^{\prime} \subseteq \eta$ (Int $\left.H\right)$. We show that $D^{\prime}$ separates the $\eta\left(J_{i}\right)$ 's nontrivially in $\eta(H)$, so that $\eta^{-1}\left(D^{\prime}\right) \cap H$ can be taken as $D$.

To do this, construct a 3-manifold $M^{*}$ containing $\eta(H)$ by attaching 3-cells to $\eta(H)$ along each annulus $\eta\left(N\left(J_{i}, \mathrm{Bd} H\right)\right)$. van Kampen's Theorem and the fact that the $\eta\left(J_{i}\right)$ 's generate the fundamental group of $\eta(H)$ imply that $\pi_{1}\left(M^{*}\right)$ is trivial and hence $\mathrm{Bd} M^{*}$ is a 2-sphere. Since $D^{\prime} \cap \mathrm{Bd} M^{*}=\mathrm{Bd} D^{\prime}, D^{\prime}$ separates $M^{*}$ and since 
$D^{\prime} \subseteq \eta(H), D^{\prime}$ separates $\eta(H)$ also. If the $\eta\left(J_{i}\right)$ 's were separated trivially in $\eta(H)$ by $D^{\prime}$, then $\mathrm{Bd} D^{\prime}$ would bound a 2-cell on $\eta(\mathrm{Bd} H)$, in contradiction to a previous observation. This completes the proof of the first assertion.

The proof of the Theorem now proceeds by an easy inductive verification of :

$\mathrm{P}(n)$ : Let $H$ and $H^{*}$ be solid tori, $g(H)=n=g\left(H^{*}\right)$, and let $J_{1}, \cdots, J_{n}$ and $J_{1}^{*}, \cdots, J_{n}^{*}$ be polyhedral simple closed curves which generate $\pi_{1}(H)$ and $\pi_{1}\left(H^{*}\right)$, respectively. Then there is a piecewise linear homeomorphism of $H$ onto $H^{*}$ which throws the elements of the first collection onto those of the second.

$\mathrm{P}(1)$ is known. We prove $\mathrm{P}(n)$ for $n \geqq 2$ assuming $\mathrm{P}(i)$ for $1 \leqq i<n$. We assume without loss of generality that $J_{1}^{*}, \cdots, J_{n}^{*}$ are curves "nicely" located on a standard solid torus $H^{*}$ of genus $n$ in $E^{3}$. As shown above, there is a polyhedral 2-cell $D$ such that $D \cap B d H$ $=\operatorname{Bd} D, D \cap \bigcup_{i=1}^{n} J_{i}=\varnothing$, and $D$ separates the $J_{i}$ 's nontrivially in $H$.

By the Lemma, $H=H_{0} \cup H_{1}$ where $H_{0}, H_{1}$ are solid tori and $H_{0} \cap H_{1}=D$. Assume the notation chosen so that the first $k$ of the $J_{i}$ 's lie in $H_{0}$ and the remaining $(n-k)$ in $H_{1}(1 \leqq k \leqq n-1)$. Because we dictated the location of the $J_{i}^{*}$ 's on $H^{*}$, we can write $H^{*}=H_{0}{ }^{*} \cup H_{1}^{*}$ with $H_{0}^{*} \cap H_{1}^{*}=D^{*}$ ( $D^{*}$ is a 2 -cell and $H_{i}^{*}$ is a solid torus), the first $k$ of the $J_{i}^{* \prime}$ in $H_{0}^{*}$, and the remainder in $H_{1}^{*}$. Also $g\left(H_{0}^{*}\right)=k$ and $g\left(H_{1}^{*}\right)=n-k$.

Since $H_{0}, H_{1}$ are retracts of $H, J_{1}, \cdots, J_{k}$ generate $\pi_{1}\left(H_{0}\right)$ and $J_{k+1}, \cdots, J_{n}$ generate $\pi_{1}\left(H_{1}\right)$. Since $g\left(H_{0}\right) \leqq k, g\left(H_{1}\right) \leqq n-k$ (the fundamental group of a solid torus is free of rank equal to its genus), and $g\left(H_{0}\right)+g\left(H_{1}\right)=n$, we have $g\left(H_{0}\right)=k$ and $g\left(H_{1}\right)=n-k$.

Let $N(D, H)$ and $N\left(D^{*}, H^{*}\right)$ be regular neighborhoods of $D$ and $D^{*}$ in $H$ and $H^{*}$, respectively. These neighborhoods are 3-cells and are chosen to be disjoint from the generating curves and so that $N(D, H) \cap \mathrm{Bd} H$ is a regular neighborhood of $\mathrm{Bd} D$ in $\mathrm{Bd} H$, and similarly for $N\left(D^{*}, H^{*}\right)$. Denote by $M_{i}$ and $M_{i}^{*}(i=0,1)$ the closures of the components of $H-N(D, H)$ and $H^{*}-N\left(D^{*}, H^{*}\right)$, respectively, such that $M_{i} \subseteq H_{i}$ and $M_{i}^{*} \subseteq H_{i}{ }^{*}$.

The pair $\left(M_{i}, M_{i}^{*}\right)$ satisfies (with respect to the appropriate generating curves) the hypothesis of $\mathrm{P}(k)$ when $i=0$ and that of $\mathrm{P}(n-k)$ when $i=1$ (since the same is true of $\left(H_{i}, H_{i}^{*}\right)$ ). Let $h_{0}$ be a homeomorphism of $M_{0}$ onto $M_{0}^{*}$ taking $J_{1}, \cdots, J_{k}$ onto $J_{1}^{*}, \cdots, J_{k}^{*}$ in some order. Let $h_{1}$ be a homeomorphism of $M_{1}$ onto $M_{1}^{*}$ taking $J_{k+1}, \cdots, J_{n}$ onto $J_{k+1}^{*}, \cdots, J_{n}^{*}$ in some order. We may now adjust $h_{0}$ so as to map the 2-cell $N(D, H) \cap M_{0}$ onto the 2-cell $N\left(D^{*}, H^{*}\right)$ 
$\cap M_{0}^{*}$ by following $h_{0}$ by an autohomeomorphism of $M_{0}^{*}$ which throws $h_{0}\left(N(D, H) \cap M_{0}\right)$ onto $N\left(D^{*}, H^{*}\right) \cap M_{0}^{*}$ and is the identity on $J_{1}^{*}, \cdots, J_{k}^{*}$. Similarly for $h_{1}$.

We also wish to have $h_{0}$ and $h_{1}$ map the two boundary components of $N(D, H) \cap \mathrm{Bd} H$ onto the two boundary components of $N\left(D^{*}, H^{*}\right)$ $\cap \mathrm{Bd} H^{*}$ in the same sense. If this is not the case, follow $h_{0}$ with another autohomeomorphism of $M_{0}^{*}$ which reverses the orientation of the curve $M_{0}^{*} \cap N\left(D^{*}, H^{*}\right) \cap \mathrm{Bd} H^{*}$ and maps the curves $J_{1}^{*}, \cdots, J_{k}^{*}$ among themselves. With this done, we can find a homeomorphism $h$ of $H$-Int $N(D, H)$ onto $H^{*}-\operatorname{Int} N\left(D^{*}, H^{*}\right)$ which extends both $h_{0}$ and $h_{1}$. Finally, $h$ extends to map Int $N(D, H)$ onto Int $N\left(D^{*}, H^{*}\right)$ homeomorphically and this completes the proof.

Added in proof. Essentially, the same result has been obtained (by different methods) by Heiner Zieschang in Abh. Math. Sem. Univ. Hamburg 25 (1962), 231-250 (see Satz 2, p. 239). The paper by Zieschang appeared after this paper was accepted. I wish to thank C. D. Papakyriakopoulos for bringing this to my attention.

\section{REFERENCES}

1. C. D. Papakyriakopoulos, On solid tori, Proc. London Math. Soc. 7 (1957), 281-299.

2. - On Dehn's Lemma and the asphericity of knots, Ann. of Math. (2) 66 (1957), 1-26.

3. E. van Kampen, On the connection between the fundamental groups of some related spaces, Amer. J. Math. 55 (1933), 261-267.

4. J. H. C. Whitehead, Simplicial spaces, nuclei and m-groups, Proc. London Math. Soc. 45 (1939), 243-327.

Florida State University 